\section{Avaliação da incompletude da variável escolaridade materna nos registros das Declarações de Nascidos Vivos nas capitais brasileiras - 1996 a 2013}

\author{
Assessment of the incompleteness of the \\ maternal schooling variable in Live Birth \\ Certificate databases in Brazilian state \\ capitals, 1996-2013
}

\section{Evaluación de la incompletitud de la variable escolaridad materna en los registros de las Declaraciones de Nacidos Vivos en capitales brasileñas, de 1996 a 2013}

Sonia Silvestrin 1,2

Viviane Costa de Souza Buriol 2,3

Clécio Homrich da Silva 2

Marcelo Zubaran Goldani 2

doi: 10.1590/0102-311X00039217

\section{Resumo}

O presente artigo avaliou a qualidade de preenchimento da variável escolaridade da mãe nas capitais brasileiras e sua distribuição regional, por intermédio do Sistema de Informações sobre Nascidos Vivos (SINASC), processado pela Declaração de Nascido Vivo (DNV). Foi realizado um estudo descritivo de uma série temporal no período de 1996 a 2013, com um total de 12.062.064 nascimentos, dos quais 11.442.494 (94,86\%) possuíam informação válida para a variável escolaridade da mãe. Os resultados foram calculados por número de incompletude da variável para cada 1.000 nascidos vivos e foi avaliada a tendência por meio do software Jointpoint (versão 4.3.1). A análise regional demonstrou que a Região Sul apresentou uma tendência de redução da incompletude da escolaridade materna, mantida no período do estudo, em todas as suas capitais. Igualmente, de forma geral, a maior parte das outras capitais do país também evidenciou uma melhora na completude da variável. Entretanto, verificaram-se diferentes tendências, com algumas capitais, inclusive, apresentando uma maior incompletude ao final do período, quando comparado ao seu início. O SINASC demonstrou ser um instrumento valioso nas informações sobre as mães e seus recém-nascidos juntamente com as condições de parto e nascimento no país. Particularmente, a escolaridade materna, considerada um fator importante sobre os desfechos obstétricos e neonatais, é uma variável que permite a elaboração e avaliação das políticas e ações na área da saúde materno-infantil. Assim, alcançar a sua máxima completude requer um esforço conjunto, dos profissionais e gestores, garantindo a credibilidade dessas informações.

Declaração de Nascimento; Escolaridade; Sistemas de Informação

\author{
Correspondência \\ S. Silvestrin \\ Secretaria Municipal de Saúde de Porto Alegre. \\ Av. João Pessoa 325, Porto Alegre, RS 90040-000, Brasil. \\ soniasilvestrin@hotmail.com \\ 1 Secretaria Municipal de Saúde de Porto Alegre, Porto Alegre, \\ Brasil. \\ 2 Universidade Federal do Rio Grande do Sul, Porto Alegre, \\ Brasil. \\ 3 Centro Universitário Ritter dos Reis, Porto Alegre, Brasil.
}




\section{Introdução}

Uma das finalidades das informações em saúde é fornecer subsídios para ações adequadas no setor em que elas estão relacionadas ${ }^{1}$. Assim, as estatísticas vitais sobre os nascimentos são essenciais para o reconhecimento do perfil epidemiológico, o planejamento e a avaliação das ações de saúde materna e infantil 2. Possibilitam a avaliação de diversos indicadores, como o cálculo do coeficiente de mortalidade infantil e o percentual de recém-nascidos de baixo peso, dentre outros 3 .

Os registros de nascimentos no Brasil, até o início da década de 1990, estiveram baseados exclusivamente no Sistema de Registro Civil, que compreendia apenas os que eram informados em cartório, com níveis variáveis de sub-registro, considerando as diferentes regiões do país. Em razão da importância em conhecer as informações relacionadas aos nascidos vivos para as estatísticas de saúde, epidemiologia e demografia, foi implantado o Sistema de Informações sobre Nascidos Vivos (SINASC) 4. Lançado oficialmente pelo Ministério da Saúde em 1990, está sob a responsabilidade das Secretarias Municipais e Estaduais de Saúde e visa ao registro de informações sobre os nascimentos vivos em âmbito nacional, com a coleta de dados que permitem conhecer o perfil epidemiológico dos nascimentos, representando uma fonte de informação relevante para as investigações na área de saúde materno-infantil 4,5,6.

O desenvolvimento do SINASC não ocorreu de forma homogênea em todo país. Sua implantação aconteceu de maneira gradual entre as Unidades da Federação (UF), de forma que seus dados nacionais foram divulgados apenas a partir de 1994 2. Atualmente, está estabelecido em todos os estados brasileiros e as informações são divulgadas pela página eletrônica do Departamento de Informática do SUS (DATASUS; http://datasus.gov.br) em um prazo de, no máximo, dois anos após a ocorrência do nascimento 2,4 .

Conforme referido pelo Ministério da Saúde, a avaliação do SINASC tem mostrado que esse sistema de informação ampliou a cobertura dos eventos estimados em todo o país, de $90 \%$ em 2005 , para 96\% em 2009. Porém, valores inferiores a 90\% ainda são observados em algumas Unidades Federativas 7 .

As informações do SINASC estão baseadas na Declaração de Nascido Vivo (DNV), documento oficial e padronizado para todo o país que é de emissão obrigatória nos hospitais e outras instituições de saúde em que são realizados partos. Os cartórios de Registro Civil também apresentam tal obrigatoriedade quando relacionada com os partos ocorridos no domicílio ${ }^{8}$. O fluxo de encaminhamento de tais declarações, bem como as normas quanto ao seu preenchimento e processamento das informações, é definido pelo Ministério da Saúde e estão contempladas nos manuais de procedimentos e preenchimentos da DNV 9.

A DNV passou por várias modificações desde a implantação do SINASC com a inclusão, supressão e qualificação das suas diversas variáveis. A versão atual é de 2010 e contempla dados relacionados à mãe, ao pré-natal, ao parto e ao recém-nascido, proporcionando informações como: idade e escolaridade da mãe, parição, duração da gestação, número de consultas de pré-natal, tipo de parto, índice de Apgar, peso ao nascer, estabelecimento onde ocorreu o parto e município de origem da mãe 2,5,6. Desse modo, o SINASC é atualmente um instrumento essencial para planejar ações na área materno-infantil no país, pois contém informações relevantes acerca da gestação, do parto e do nascimento. No entanto, à medida que a cobertura do sistema aumenta, surge a preocupação com a melhora da fidedignidade das informações e da completude das variáveis que conferem credibilidade ao sistema e permitem confiabilidade sobre os resultados obtidos nas análises de suas variáveis e para o planejamento nos diferentes níveis de complexidade dos serviços de saúde, nas diferentes regiões do país 10.

Nessa perspectiva, a escolaridade da mãe, um importante indicador da inserção social materna, tem sido relacionada com os desfechos da gravidez, do parto e do recém-nascido 11,12,13,14. Essa variável compreende a percepção, as atitudes e a prática das mulheres, repercutindo no acesso, acompanhamento e supervisão de saúde, nos cuidados na gestação e na redução de seus eventos adversos 11,13. Dessa forma, conhecer o perfil de escolaridade materna mostra fundamental relevância para programar o desenvolvimento de ações e intervenções na área de saúde materno-infantil. Sendo assim, torna-se necessário verificar a consistência qualitativa e quantitativa dessa variável contida no SINASC, de modo que possa traduzir de forma fidedigna a realidade da condição educacional da mãe. 
O presente estudo objetivou avaliar a qualidade de preenchimento da variável escolaridade da mãe no SINASC, em todos os nascimentos ocorridos nas capitais brasileiras no período de 1996 a 2013, considerando sua representatividade da condição socioeconômica da mãe e sua relevância nos desfechos da gravidez e do parto.

\section{Métodos}

Foi realizado estudo descritivo de série temporal em que foram levados em conta todos os registros dos nascidos vivos de partos que ocorreram nas capitais brasileiras no período de 1996 a 2013, informados na base de dados do SINASC por intermédio das DNV. As capitais foram escolhidas para verificar a completude dos dados por possuírem mais estrutura logística de pessoal e serviços, adequarem-se mais rapidamente às normativas nacionais e balizarem as ações dos outros municípios de menor porte dentro do seu estado.

A página eletrônica do DATASUS foi acessada, por intermédio do programa TabWin (DATASUS. http://portal.saude.gov.br/portal/se/datasus/area.cfm?id_area=732), sendo os arquivos convertidos do formato DBC para DBF para, então, serem extraídas as informações das 27 capitais brasileiras. Seu processamento e análise foi desenvolvido no IBM SPSS versão 18.0. (IBM Corp., Armonk, Estados Unidos).

Num segundo momento, foi processado um novo banco de dados com as informações relativas à variável escolaridade da mãe e calculada a incompletude ano a ano, para cada uma das capitais, agrupadas por região geográfica (Norte, Nordeste, Centro-oeste, Sudeste e Sul). Para a melhor interpretação dos resultados foi calculado, anualmente, o número de perdas da variável para cada mil nascidos vivos, o que foi chamado de "coeficiente de incompletude".

Para analisar a tendência temporal da incompletude da variável, em cada uma das capitais brasileiras, foram calculadas as variações percentuais anuais (APC), utilizando o software Joinpoint Regression Program, versão 4.3.1 (National Cancer Institute; https://surveillance.cancer.gov/joinpoint/). Essa análise objetivou a identificação de possíveis joinpoints em que ocorra uma mudança na tendência. Baseado num modelo com até 3 pontos de mudança foi testado se os vários segmentos da reta permitem uma melhor avaliação de tendência do que uma reta única 15,16. Esse modelo foi ajustado com a APC, fundamentado na tendência de cada segmento, estimando se os valores foram estatisticamente significativos com um nível de $95 \%$ de confiança. Os testes de significância utilizados basearam-se no método de permutação de Monte Carlo e no cálculo da APC do logaritmo da razão 17.

Destaca-se que, no ano de 1999, houve uma incompletude elevada em grande parte das capitais, diferindo de seu comportamento histórico, possivelmente relacionado à alteração no formulário da DNV ocorrido naquele ano, quando a variável escolaridade da mãe foi modificada 18,19. Dessa maneira, pode ter havido dificuldade na importação das informações para a base de dados do DATASUS, ocasionando perdas em diversas capitais das diferentes regiões, com o seu reestabelecimento nos anos posteriores. Nessa perspectiva, considerando as perdas elevadas e no intuito de não prejudicar a análise temporal proposta pelo presente estudo, o ano de 1999 foi excluído das análises.

O estudo foi submetido e aprovado pelo Comitê de Ética e Pesquisa do Hospital de Clínicas de Porto Alegre (HCPA), com o parecer no 16-0338 e não recebeu financiamento. Conforme a Resolução no 466/2012 do Conselho Nacional de Saúde, não há riscos para os sujeitos de pesquisa e não há conflitos de interesse.

\section{Resultados}

No período de 1996 a 2013, foram registrados 12.062 .064 de nascidos vivos nas capitais brasileiras que representaram 22,3\% dos nascimentos ocorridos em todo o país no período. Desses, 11.442.494 $(94,86 \%)$ possuíam informação válida para a variável escolaridade da mãe, enquanto 619.570 (5,14\%) não apresentavam essa informação disponível, considerando os campos em branco ou ignorado.

Foi encontrada uma variabilidade no preenchimento da variável escolaridade da mãe, tendo em vista a capital avaliada e o transcorrer dos anos da série temporal. Identificaram-se capitais em que a incompletude era elevada no início do período e foi, gradativamente, sendo reduzida até alcançar 
números muito baixos ao final, como verificado em São Paulo e Campo Grande (Mato Grosso do Sul). Por outro lado, verificaram-se capitais onde a incompletude da informação era elevada e permaneceu nessa condição durante todo o período, como constatado em Porto Velho (Rondônia) e Fortaleza (Ceará). Em outras ainda sucedeu redução e após, novamente, elevação da incompletude no final do período avaliado como evidenciado na capital Macapá (Amapá).

A Tabela 1 mostra o número de informações incompletas para a variável escolaridade materna a cada mil nascidos vivos, referente aos campos em branco ou ignorado, de acordo com a capital de ocorrência do parto, agrupadas por região geográfica. Observou-se uma variabilidade entre as capitais e não ocorreu tendência uniforme na redução da incompletude da variável, uma vez que aconteceu oscilação. Entretanto, de forma geral, a maior parte das capitais demonstrou uma qualificação no preenchimento da variável no período estudado.

Analisando as capitais nas regiões geográficas a que pertencem se observou que na Região Norte, as capitais Manaus (Amazonas), Porto Velho, Palmas (Tocantins), Rio Branco (Acre) e Belém (Pará) apresentavam uma incompletude elevada para a variável nos anos iniciais da série temporal. No entanto, gradativamente, a maior parte delas qualificou o preenchimento da informação com uma expressiva redução da incompletude. Manaus reduziu de 122,0/1.000 em 1996 para 10,2/1.000 em 2013; Palmas, de 40,3/1.000 para 7,8/1.000; e Belém, de 33,4/1.000 para 4,2/1.000, respectivamente. A capital Porto Velho, como já referido, mostrou incompletude elevada e continuou nessa condição durante todo o período, atingindo em 2007, 158,6/1.000, o maior coeficiente mostrado na região, considerando a série temporal avaliada. Na capital Boa Vista (Roraima), a incompletude que já era pequena foi reduzida ainda mais e, em Macapá, a incompletude se apresentava pequena no início da série, porém, no decorrer do período, demonstrou uma elevação, especialmente a partir de 2009 $(18,5 / 1.000)$.

A Região Nordeste também mostrou perdas elevadas no início da série nas capitais Aracaju (Sergipe), João Pessoa (Paraíba) e São Luís (Maranhão) com 769,4/1.000, 419,6/1.000 e 367,6/1.000, respectivamente, contudo com melhora gradativa e persistente da incompletude que, ao final do período foi de 0,4/1.000, 18,7/1.000 e 17,4/1.000, respectivamente. Essa situação também pôde ser constatada em Recife (Pernambuco), Maceió (Alagoas) e Salvador (Bahia), que apresentavam incompletude menor no início da série e, no decorrer dos anos, mostraram melhora no preenchimento da variável. Diferentemente, as capitais Teresina (Piauí) e Natal (Rio Grande do Norte) demonstraram a menor incompletude da região, inicialmente, com temporária redução das perdas e após, novamente, com um aumento no final do período. A capital Fortaleza mostrou perdas elevadas no início que seguiram aumentando até 2007 e, mesmo sofrendo redução após esse ano, foi a capital com maior incompletude do Nordeste entre os anos de 2011 e 2013 e a maior de todas as regiões no ano de 2013 (56,5/1.000).

Os resultados observados na Região Centro-oeste mostraram que Campo Grande era a capital com maior incompletude no início do período com 296,8/1.000, mas, nos anos seguintes, houve uma redução persistente das perdas e, ao final do período analisado, foi verificado o menor coeficiente da região, 0,2/1.000. Goiânia (Goiás) e Brasília (Distrito Federal), por sua vez, foram as capitais do Centro-oeste com a menor qualidade de preenchimento da variável em todos os anos avaliados e, apesar de mostrarem alternância entre redução e elevação das perdas no período, não sinalizaram para uma qualificação no preenchimento da variável, pois mantiveram os maiores coeficientes das capitais no país chegando ao final do período a 22,0/1.000 e 30,3/1.000, respectivamente.

$\mathrm{Na}$ região Sudeste verificou-se um desempenho distinto da incompletude entre suas capitais. Enquanto Belo Horizonte (Minas Gerais) e São Paulo mostraram redução gradativa e persistente das perdas de informações atingindo, ao final do período, as menores taxas da região (2,0 e 1,3/1.000, respectivamente), Vitória (Espírito Santo) mostrou um acréscimo. De forma peculiar, o Rio de Janeiro mostrou uma redução, porém, de 2011 a 2013, a capital carioca mostrou aumento da incompletude e, neste último ano foi a capital da Região Sudeste com o maior coeficiente (35,9/1.000).

A incompletude na Região Sul mostrou redução em todas as capitais, com perdas inexpressivas em 2013. Curitiba (Paraná) e Porto Alegre (Rio Grande do Sul) mostraram redução gradativa e persistente das perdas e encerraram o período avaliado com uma incompletude de 0,5/1.000 e 2,3/1.000, respectivamente. A capital Florianópolis (Santa Catarina) mostrou a maior incompletude do Sul no início da série temporal e se manteve em tal condição durante todo o período analisado, com redução no decorrer dos anos. 
Coeficiente de incompletude (número de informações em branco ou ignorado/por 1.000 nascidos vivos) para a variável escolaridade da mãe nas capitais brasileiras no período de 1996-2013 *.

\begin{tabular}{|c|c|c|c|c|c|c|c|c|c|c|c|c|c|c|c|c|c|}
\hline Regiões/Capitais & 1996 & 1997 & 1998 & 2000 & 2001 & 2002 & 2003 & 2004 & 2005 & 2006 & 2007 & 2008 & 2009 & 2010 & 2011 & 2012 & 2013 \\
\hline \multicolumn{18}{|l|}{ Região Norte } \\
\hline Porto Velho & 52,0 & 100,5 & 86,2 & 92,4 & 91,2 & 89,5 & 84,4 & 79,7 & 115,8 & 88,2 & 158,6 & 122,1 & 114,0 & 112,4 & 78,5 & 56,2 & 54,9 \\
\hline Rio Branco & 34,2 & 23,9 & 26,8 & 11,7 & 15,1 & 17,6 & 24,8 & 1,9 & 0,8 & 14,9 & 13,4 & 10,3 & 9,8 & 4,4 & 9,4 & 14,2 & 13,4 \\
\hline Manaus & 122,0 & 88,9 & 71,3 & 99,0 & 63,2 & 17,9 & 14,3 & 19,8 & 18,9 & 4,8 & 4,7 & 6,5 & 5,0 & 5,4 & 7,4 & 7,0 & 10,2 \\
\hline Boa Vista & 8,9 & 3,4 & 6,0 & 8,0 & 27,2 & 0,5 & 0,2 & 0,0 & 0,5 & 1,7 & 0,8 & 1,0 & 0,0 & 0,8 & 1,3 & 0,6 & 0,6 \\
\hline Belém & 33,4 & 30,9 & 30,8 & 18,0 & 14,2 & 0,0 & 0,0 & 0,0 & 0,5 & 1,5 & 2,2 & 2,3 & 1,0 & 0,5 & 1,6 & 2,2 & 4,2 \\
\hline Macapá & 5,9 & 6,7 & 22,7 & 26,9 & 20,0 & 19,8 & 10,2 & 2,6 & 0,2 & 0,2 & 0,3 & 2,8 & 18,5 & 16,3 & 12,9 & 10,3 & 14,9 \\
\hline Palmas & 40,3 & 27,2 & 16,8 & 10,4 & 9,4 & 2,3 & 0,5 & 0,3 & 2,4 & 5,7 & 14,1 & 7,0 & 4,3 & 5,0 & 4,8 & 7,2 & 7,8 \\
\hline \multicolumn{18}{|l|}{ Região Nordeste } \\
\hline São Luís & 367,6 & 189,9 & 228,0 & 44,1 & 51,6 & 80,1 & 103,7 & 40,8 & 44,3 & 34,9 & 63,9 & 58,9 & 53,5 & 47,8 & 18,7 & 17,9 & 17,4 \\
\hline Teresina & 10,7 & 8,6 & 6,8 & 20,3 & 5,1 & 6,5 & 6,2 & 8,3 & 11,6 & 11,2 & 14,7 & 19,1 & 28,3 & 10,4 & 25,7 & 33,8 & 25,8 \\
\hline Fortaleza & 70,3 & 64,5 & 66,7 & 141,2 & 106,8 & 91,8 & 71,1 & 69,8 & 81,3 & 85,2 & 72,4 & 22,8 & 15,7 & 21,5 & 37,6 & 53,1 & 56,5 \\
\hline Natal & 21,6 & 15,0 & 25,5 & 10,0 & 4,6 & 5,7 & 3,1 & 2,5 & 4,8 & 5,3 & 6,9 & 4,0 & 3,9 & 12,0 & 15,5 & 14,5 & 18,7 \\
\hline João Pessoa & 419,6 & 187,7 & 283,2 & 17,6 & 11,1 & 13,1 & 6,8 & 6,6 & 7,9 & 7,1 & 5,7 & 4,1 & 3,2 & 3,1 & 6,3 & 5,0 & 18,7 \\
\hline Recife & 33,4 & 20,5 & 16,2 & 20,0 & 19,3 & 25,9 & 31,7 & 29,1 & 18,9 & 14,0 & 3,8 & 2,0 & 2,8 & 1,2 & 2,8 & 2,9 & 5,5 \\
\hline Maceió & 27,2 & 23,5 & 32,7 & 16,4 & 29,6 & 37,4 & 36,7 & 36,6 & 17,6 & 10,8 & 5,8 & 4,2 & 4,0 & 4,3 & 7,2 & 5,5 & 6,0 \\
\hline Aracaju & 769,4 & 792,2 & 830,3 & 55,3 & 35,3 & 19,6 & 23,3 & 26,0 & 19,8 & 7,0 & 0,9 & 0,6 & 0,6 & 0,0 & 0,3 & 0,2 & 0,4 \\
\hline Salvador & 65,8 & 47,6 & 53,9 & 76,1 & 67,0 & 49,0 & 33,4 & 18,6 & 15,6 & 9,4 & 2,9 & 3,6 & 4,5 & 5,2 & 11,1 & 12,6 & 9,8 \\
\hline \multicolumn{18}{|l|}{ Região Centro-oeste } \\
\hline Campo Grande & 296,8 & 14,1 & 15,1 & 5,7 & 2,9 & 2,8 & 0,6 & 0,7 & 0,3 & 0,8 & 0,1 & 0,0 & 0,2 & 0,3 & 0,5 & 0,8 & 0,2 \\
\hline Cuiabá & 67,7 & 40,0 & 20,2 & 7,0 & 5,3 & 5,7 & 13,5 & 1,4 & 0,6 & 0,0 & 0,0 & 0,0 & 0,4 & 0,0 & 1,8 & 2,2 & 2,4 \\
\hline Goiânia & 34,9 & 58,4 & 49,9 & 103,4 & 87,8 & 105 & 97,0 & 93,1 & 84 & 53,3 & 29,7 & 32,9 & 27,2 & 24,6 & 29,4 & 31,1 & 22,0 \\
\hline Brasília & 35,5 & 33,5 & 39,8 & 17,9 & 23,2 & 19,0 & 20,5 & 11,2 & 14,9 & 35,6 & 12,1 & 26,5 & 32,4 & 16,9 & 24,0 & 32,3 & 30,3 \\
\hline \multicolumn{18}{|l|}{ Região Sudeste } \\
\hline Belo Horizonte & 46,9 & 22,4 & 23,7 & 20,8 & 14,4 & 15,3 & 12,0 & 9,0 & 4,2 & 3,7 & 4,0 & 3,1 & 3,1 & 3,3 & 3,2 & 4,1 & 2,0 \\
\hline Vitória & 4,6 & 1,8 & 7,5 & 8,3 & 9,8 & 4,5 & 11,6 & 12,0 & 23,6 & 35,6 & 42,8 & 33,2 & 12,2 & 15,4 & 28,1 & 28,8 & 20,6 \\
\hline Rio de Janeiro & 52,0 & 43,6 & 47,4 & 42,3 & 30,4 & 29,5 & 27,7 & 23,8 & 20,1 & 14,3 & 17,3 & 30,1 & 21,1 & 11,4 & 14,0 & 22,0 & 35,9 \\
\hline São Paulo & 920,6 & 207,5 & 212,4 & 112,5 & 63,0 & 51,2 & 50,3 & 30,1 & 30,7 & 12,0 & 8,3 & 4,8 & 2,1 & 0,8 & 0,8 & 2,0 & 1,3 \\
\hline \multicolumn{18}{|l|}{ Região Sul } \\
\hline Curitiba & 13,7 & 16,0 & 10,3 & 25,4 & 14,2 & 9,0 & 3,7 & 1,1 & 0,5 & 0,7 & 0,9 & 0,6 & 2,7 & 1,1 & 1,0 & 1,1 & 0,5 \\
\hline Florianópolis & 83,3 & 82,0 & 32,7 & 53,5 & 69,5 & 31,9 & 33,8 & 20,3 & 49,8 & 31,5 & 3,5 & 6,2 & 25,0 & 4,4 & 14,4 & 10,3 & 3,2 \\
\hline Porto Alegre & 7,7 & 8,0 & 6,3 & 29,9 & 8,7 & 7,9 & 7,2 & 3,0 & 3,3 & 4,7 & 1,2 & 1,5 & 1,5 & 0,5 & 3,4 & 4,4 & 2,3 \\
\hline
\end{tabular}

Fonte: Departamento de Informática do SUS. Sistema de Informações sobre Nascidos Vivos (SINASC).

* Excluído o ano de 1999 por apresentar números elevados de incompletude.

A Tabela 2 mostra a APC da incompletude da variável escolaridade da mãe explicando as tendências em cada uma das capitais, no período de 1996 a 2013.

Verificou-se que as capitais, Rio Branco, Manaus, Belém, São Luís, Campo Grande, Cuiabá, Belo Horizonte, Rio de Janeiro, São Paulo, Florianópolis e Porto Alegre mostraram tendência de redução da incompletude em todo o período analisado, sem mudança na inclinação das retas, enquanto as capitais Teresina e Vitória mostraram tendência de aumento em todo o período analisado. São Paulo e Aracaju verificaram a maior redução de incompletude comparativamente às demais capitais, com ponto de inflexão em 1998.

Destacaram-se as capitais Palmas, Fortaleza, Natal, Salvador e Brasília, onde se verificou redução da incompletude em alguns períodos durante a série temporal, porém não de forma sustentada e, ao final do período avaliado, Fortaleza apresentou a maior coeficiente de incompletude. A capital Porto Velho mostrou uma tendência de redução após 2009 (APC = -20,9; IC95\%: -35,6; -2,3), no entanto o coeficiente de perdas ainda permaneceu o mais elevado da Região Norte. 


\section{Tabela 2}

Variação percentual anual (annual percentage change - APC) da incompletude da variável escolaridade da mãe informada no Sistema de Informações sobre Nascidos Vivos (SINASC) nas capitais brasileiras no período de 1996-2013.

\begin{tabular}{|c|c|c|c|c|c|c|c|c|}
\hline Regiões/Capitais & APC1 & IC95\% & Joinpoint & APC2 & IC95\% & Joinpoint & APC3 & IC95\% \\
\hline \multicolumn{9}{|l|}{ Região Norte } \\
\hline Porto Velho & 4,2 & 0,$9 ; 7,6$ & 2009 & $-20,7$ & $-35,6 ;-2,3$ & & & \\
\hline Rio Branco & $-6,2$ & $-9,6 ;-2,7$ & & & & & & \\
\hline Manaus & $-17,8$ & $-22,1 ;-13,3$ & & & & & & \\
\hline Boa Vista & 29,3 & 8,$9 ; 53,5$ & 2001 & -30.4 & $-40,8 ;-18,1$ & & & \\
\hline Belém & $-17,4$ & $-21,4 ;-13,2$ & & & & & & \\
\hline Macapá & $-1,8$ & $-6,9 ; 3,5$ & & & & & & \\
\hline Palmas & $-27,6$ & $-39,6 ;-13,2$ & 2002 & 3.0 & $-9,9 ; 17,7$ & & & \\
\hline \multicolumn{9}{|l|}{ Região Nordeste } \\
\hline São Luís & $-30,3$ & $-45,2 ;-11,3$ & 2000 & -7.6 & $-14,4 ;-0,3$ & & & \\
\hline Teresina & 7,6 & 3,$3 ; 12,0$ & & & & & & \\
\hline Fortaleza & 22,6 & $-23,1 ; 95,6$ & 2000 & -12.3 & $-20,0 ;-3,8$ & 2010 & 18,5 & $-33,1 ; 110,1$ \\
\hline Natal & $-22,7$ & $-32,0 ;-12,1$ & 2004 & 20.8 & 7,$9 ; 35,3$ & & & \\
\hline João Pessoa & $-37,7$ & $-47,7 ;-25,9$ & 2005 & -2.2 & $-41,1 ; 62,4$ & & & \\
\hline Recife & $-0,1$ & $-8,6 ; 9,2$ & 2004 & -23.6 & $-35,1 ;-10,0$ & & & \\
\hline Maceió & 4,5 & $-6,3 ; 16,7$ & 2003 & -21.5 & $-29,8 ;-12,2$ & & & \\
\hline Aracaju & 3,0 & $-4,1 ; 10,7$ & 1998 & $-50,5$ & $-55,6 ;-44,8$ & & & \\
\hline Salvador & 5,0 & $-4,3 ; 15,2$ & 2001 & -33.8 & $-43,7 ;-22,2$ & 2008 & 28,0 & $-6,9 ; 75,9$ \\
\hline \multicolumn{9}{|l|}{ Região Centro-oeste } \\
\hline Campo Grande & $-83,5$ & $-88,5 ;-76,4$ & 1998 & -22.2 & $-29,0 ;-14,8$ & & & \\
\hline Cuiabá & $-22,8$ & $-28,4 ;-16,7$ & & & & & & \\
\hline Goiânia & 17,7 & 5,$4 ; 31,3$ & 2002 & -15.1 & $-19,3 ;-10,7$ & & & \\
\hline Brasília & $-11,7$ & $-24,6 ; 3,5$ & 2002 & 4.5 & $-2,6 ; 12,0$ & & & \\
\hline \multicolumn{9}{|l|}{ Região Sudeste } \\
\hline Belo Horizonte & $-16,6$ & $-19,1 ;-14,0$ & & & & & & \\
\hline Vitória & 9,3 & 2,$2 ; 16,9$ & & & & & & \\
\hline Rio de Janeiro & $-5,0$ & $-7,6 ;-2,3$ & & & & & & \\
\hline São Paulo & $-57,1$ & $-63,3 ;-49,8$ & 1998 & -25.3 & $-29,4 ;-20,8$ & & & \\
\hline \multicolumn{9}{|l|}{ Região Sul } \\
\hline Curitiba & 11,2 & $-27,0 ; 69,3$ & 2000 & -26.7 & $-33,9 ;-18,7$ & & & \\
\hline Florianópolis & $-11,3$ & $-15,5 ;-6,9$ & & & & & & \\
\hline Porto Alegre & $-10,6$ & $-18,8 ;-1,7$ & & & & & & \\
\hline
\end{tabular}

IC95\%: intervalo de 95\% de confiança.

Observou-se que grande parte das capitais tem qualificado as informações referentes à variável escolaridade da mãe e que coeficientes elevados de incompletude encontrados no início da série foram sendo gradativamente reduzidos e, no ano de 2013, somente Fortaleza e Porto Velho ainda mostraram perdas superiores a 50 para cada mil nascidos vivos.

\section{Discussão}

O número de anos de estudo vem aumentando em todas as regiões do Brasil 20,21. Na última década, têm sido realizados grandes investimentos na educação básica, resultando na diminuição da taxa de analfabetismo e no acréscimo da escolarização das mulheres 22 . A média nacional de anos de estudo da população brasileira é de 7,5, mas se observam diferenças entre as regiões geográficas: Norte, 7,0 anos; Nordeste, 6,6; Centro-oeste, 8,0; Sudeste, 8,1 e Sul, 7,8 23 . 
Uma melhor escolaridade materna tem mostrado relação com a redução das taxas de baixo peso ao nascer 12,21, com o declínio das taxas de mortalidade infantil 24 e com a melhora na cobertura de prénatal 25. Por outro lado, a baixa escolaridade e as adversidades socioeconômicas e culturais são fatores associados a problemas de saúde para a mãe e para a criança, tanto quanto os custos econômicos e os problemas sociais decorrentes, como a perpetuação da pobreza, são ressaltados como importantes consequências neste cenário 26,27,28.

O reconhecimento do conjunto dos atributos maternos como as condições socioeconômicas, a idade, a escolaridade e a ocupação permitem uma maior precisão na avaliação da desigualdade social e de riscos para os recém-nascidos 5 .

Considerando a importância do nível de instrução materno nos desfechos em saúde na área materna e infantil, bem como da qualidade dos sistemas de informação em saúde em disponibilizar os dados, o presente estudo mensurou a frequência das informações, em branco ou ignorado, para a variável escolaridade da mãe e identificou que, nos dezoito anos avaliados, ela vem sendo gradativamente qualificada na quase totalidade das capitais brasileiras e a incompletude elevada da informação, como aquela identificada nos anos de 1996 a 1998, foi sendo reduzida e permaneceu em queda durante todo o período estudado. Em 2013, somente duas capitais, Fortaleza e Porto Velho, apresentaram incompletude maior de 50/1.000; esses números representaram perdas bastante reduzidas se comparadas às encontradas em diversas capitais, no início do período investigado.

Nessa mesma perspectiva, identificou-se que 14 capitais, distribuídas nas cinco regiões, mostraram incompletude menor de 10/1.000, o que pode ser considerado como excelente 5,29. Dessas, 12 mostraram persistente tendência de redução do coeficiente desde o início da série analisada, especialmente após o ano de 2000. Resultado semelhante foi encontrado por Guimarães et al. 30 no estudo que investigou a completude das variáveis em 38 municípios do Estado de Minas Gerais. Nele, os autores relataram melhora da completude de todas as variáveis relacionadas às características maternas após o ano de 2000, bem como na completude dos municípios de grande porte onde o preenchimento foi identificado como adequado para todas as variáveis relacionadas à mãe. Também, Silva et al. 10 observaram melhoria do percentual de incompletude da escolaridade da mãe na Região Nordeste, em 2009, tirante os estados da Paraíba e Bahia. Em Pernambuco, também foi verificada melhora de 12,61\% ao ano na completude dessa variável 2 .

A qualificação encontrada no preenchimento da variável pode estar relacionada a diversos fatores como o melhor acesso aos sistemas de informação mediante a incorporação das tecnologias de informação, a capacitação dos profissionais responsáveis pelo seu preenchimento e processamento dos dados e o aprimoramento do prontuário médico-hospitalar, que muitas vezes é a fonte dos dados para o preenchimento da DNV 2,30.

A presente análise da incompletude das capitais não mostrou relação com o grau de desenvolvimento socioeconômico e tecnológico porque, dentre as que mostraram os maiores coeficientes de incompletude, encontram-se cidades como Rio de Janeiro e Brasília, que têm os melhores Índices de Desenvolvimento Humano (IDH) do país. Ao contrário, outras, com menor IDH, como Campo Grande e Aracaju, mostraram as menores taxas de incompletude do país em 201331.

A incompletude da escolaridade da mãe foi caracterizada quando o campo da variável no SINASC apresentava-se em branco ou preenchido como "ignorado". Todavia, diferentes fatores podem estar relacionados à ausência da informação. Enquanto em "branco" pode ter relação com o não preenchimento do campo pelo profissional; "ignorado" pode ser pela indisponibilidade de tal informação. Nesse ponto de vista, algumas publicações referiram que uma das restrições para a melhor completude da variável foi a ausência das informações no prontuário hospitalar e, para o seu preenchimento, haveria necessidade de uma entrevista com a puérpera ou seu acompanhante, o que, muitas vezes, torna-se difícil por conta de ela não estar em condições de responder ou do seu acompanhante não ter conhecimento sobre sua escolaridade, sendo necessária a busca de outras fontes como o cartão da gestante 2,5,30.

Por outro lado, pesquisas que analisaram o fluxo de preenchimento da DNV verificaram que o mesmo se dá com diferentes profissionais (médico, enfermeiro, técnico ou auxiliar de enfermagem, escriturário e funcionários administrativos) dentro das instituições 32,33. Essa situação traz repercussões na completude das variáveis, devido à falta de conhecimento sobre a importância do seu preenchimento, uma vez que, dependendo de sua formação profissional, determinadas informações 
não são adequadamente compreendidas ou valorizadas 1,2 . Outros motivos da incompletude também foram atribuídos a erros de grafia e de transcrição das informações, bem como aos diferentes fluxos utilizados pelas instituições para o preenchimento da DNV 4,33.

Conforme relatado por Mascarenhas \& Gomes ${ }^{34}$, a obtenção da informação sobre escolaridade da mãe é complexa e, muitas vezes, não está disponível nos registros hospitalares ou é preenchida de forma incorreta, podendo afetar sua confiabilidade. Portanto, mostra-se relevante qualificar os profissionais responsáveis pelo preenchimento da DNV, ressaltando a importância das informações e avaliando possíveis problemas encontrados para que possam ser solucionados, como a revisão do método de preenchimento das variáveis, pois as dificuldades podem determinar resultados discrepantes, já que a variável é categorizada por anos de estudos concluídos e não por períodos escolares 35 . No entanto, a versão atual da DNV é mais objetiva em relação à variável escolaridade materna, com a existência de dois campos: nível de escolaridade e série concluída. Isso torna mais fácil o preenchimento da informação por parte da puérpera e do profissional responsável 36. Igualmente relevante é a necessidade de investimentos na área de infraestrutura dos serviços de saúde, de modo que possibilite a coleta e o processamento adequado das informações.

Analisando o preenchimento de outras variáveis do SINASC, verificou-se que a escolaridade materna está entre as que apresentaram a melhor completude em grande parte dos municípios estudados, juntamente com as variáveis peso ao nascer, sexo do recém-nascido, idade da mãe, duração da gestação, número de consultas de pré-natal, tipo de gravidez e tipo de parto 4,30. Diferentemente, as variáveis número de filhos nascidos vivos e mortos, raça/cor e Apgar (10 e 50 minutos) foram descritas como de preenchimento mais deficiente 2,30,34,36. A disponibilidade das informações nos prontuários hospitalares (da puérpera e do recém-nascido) ou no cartão da gestante demonstraram auxiliar na melhora da completude das variáveis na DNV 30,37.

O estudo apresentou algumas limitações porque não mediu a cobertura, que é a capacidade do sistema de informação de obter a totalidade de eventos ocorridos em uma região, num determinado período de tempo, e nem avaliou a confiabilidade dos dados, na intenção de aferir a consistência deles. Ele se deteve exclusivamente à completude da variável escolaridade da mãe.

Por outro lado, os pontos fortes desta investigação se relacionam à coleta de informações de uma longa série temporal, contemplando todas as capitais brasileiras no período proposto. Pelo seu contingente populacional, essas cidades são representativas dos demais municípios do país e permitem uma avaliação de abrangência nacional das informações sobre a escolaridade materna da população brasileira. Além disso, dentro de um processo histórico, a investigação sobre tal variável do SINASC, ao longo dos anos, permitiu uma avaliação da sua evolução temporal no período estudado, possibilitando uma observação fidedigna de sua completude. Consequentemente, forneceu subsídios para assinalar as capitais em que se faz necessário aumentar as atividades de supervisão e controle de coleta e processamento dos dados, incentivando os profissionais ao completo e correto preenchimento das variáveis 4 .

Nos últimos anos, o SINASC tem se mostrado um instrumento valioso sobre as informações das mães e seus recém-nascidos juntamente com as condições de parto e nascimento no país, permitindo a elaboração e avaliação das políticas e ações na área da saúde materno-infantil.

Assim, os resultados do presente estudo possibilitam análises locais em relação ao preenchimento da DNV, que servirão de subsídio aos gestores públicos para o aprimoramento do sistema de forma geral, bem como para o desenvolvimento de indicadores fidedignos em saúde, assim como informações sobre a escolaridade da mãe, uma variável relevante nos desfechos da gravidez, do parto e do recém-nascido.

A correta completude das variáveis do SINASC requer um esforço conjunto desde os gestores até os profissionais responsáveis pelo preenchimento nos estabelecimentos de saúde, a fim de que se alcance número cada vez menor de perdas das informações, permitindo assim a sua credibilidade. Identifica-se a necessidade de intensificar ações voltadas à orientação e sensibilização dos envolvidos na coleta dos dados com o propósito de fortalecer os diversos sistemas de informação para que sejam mais confiáveis e possam subsidiar adequadamente as tomadas de decisões 2 . Os resultados do presente estudo na quase totalidade das capitais demostraram que os profissionais responsáveis pelo preenchimento, as equipes gestoras e as instituições de saúde têm trabalhado na busca da melhoria no preenchimento das DNV 35, contribuindo para que a elaboração de políticas públicas em saúde seja 
baseada nas características e particularidades locais, diante da realidade de cada região, para torná-las mais efetivas. Permanece o desafio de qualificação das informações para as capitais que mostraram os maiores coeficientes de incompletude.

\section{Colaboradores}

S. Silvestrin contribuiu na concepção, redação do artigo, análise e interpretação dos dados, revisão crítica relevante e aprovação final. V. C. S. Buriol e M. Z. Goldani colaboraram na análise e interpretação dos dados, revisão crítica relevante e aprovação final. C. H. Silva participou na concepção, análise e interpretação dos dados, revisão crítica relevante e aprovação final.

\section{Agradecimentos}

Vânia Naomi Hirakata participou da realização da tabulação dos dados e das análises estatísticas.

\section{Referências}

1. Jorge MHPM, Laurenti R, Gotlieb SLD. Quality analysis of Brazilian vital statistics: the experience of implementing the SIM and SINASC systems. Ciênc Saúde Coletiva 2007; 12:643-54.

2. Costa JMBS, Frias PGD. Avaliação da completitude das variáveis da Declaração de Nascido Vivo de residentes em Pernambuco, Brasil, 1996 a 2005. Cad Saúde Pública 2009; 25:613-24.

3. Silva AAM, Ribeiro VS, Borba Júnior AF, Coimbra LC, Silva RA. Avaliação da qualidade dos dados do Sistema de Informações sobre Nascidos Vivos em 1997-1998. Rev Saúde Pública 2001; 35:508-14.

4. Pedraza DF. Qualidade do Sistema de Informações sobre Nascidos Vivos (Sinasc): análise crítica da literatura. Ciênc Saúde Coletiva 2012; 10:2729-37.

5. Romero DE, Cunha CB. Avaliação da qualidade das variáveis epidemiológicas e demográficas do Sistema de Informações sobre Nascidos Vivos. Cad Saúde Pública 2007; 23:701-14.

6. Paiva NS, Coeli CM, Moreno AB, Guimarães RM, Camargo JKR. Sistema de informações sobre nascidos vivos: um estudo de revisão. Ciênc Saúde Coletiva 2011; 16 Suppl 1:S121120.

7. Departamento de Análise de Situação em Saúde, Secretaria de Vigilância em Saúde, Ministério da Saúde. Saúde Brasil 2010: uma análise da situação de saúde e de evidências selecionadas de impacto de ações de vigilância em saúde. Brasília: Ministério da Saúde; 2011.

8. Brasil. Leis no 6.015 , de 31 de dezembro de 1973. Dispõe sobre os registros públicos e dá outras providências. Diário Oficial da União 1973; 31 dez.

9. Secretaria de Vigilância em Saúde, Ministério da Saúde. Manual de instruções para preenchimento da Declaração de Nascido Vivo. Brasília: Ministério da Saúde; 2011.

10. Silva RS, Oliveira CM, Ferreira DKS, Bonfim CV. Avaliação da completitude das variáveis do Sistema de Informações sobre Nascidos Vivos - SINASC - nos estados da região Nordeste do Brasil, 2000 e 2009. Epidemiol Serv Saúde 2013; 22:347-52.

11. Dai LL, Mao YY, Luo XM, Shen YP. Prenatal care in combination with maternal educational level has a synergetic effect on the risk of neonatal low birth weight: new findings in a retrospective cohort study in Kunshan City, China. PLoS One 2014; 9:e113377. 
12. Silvestrin S, Silva CH, Hirakata VN, Goldani AA, Silveira PP, Goldani MZ. Maternal education level and low birth weight: a meta-analysis. J Pediatr (Rio J.) 2013; 899:339-45.

13 Baron R, Manniën J, te Velde SJ, Klomp T, Hutton EK, Brug J. Socio-demographic inequalities across a range of health status indicators and health behaviours among pregnant women in prenatal primary care: a cross-sectional study. BMC Pregnancy Childbirth 2015; 15:261.

14. Tampah-naah A, Anzagra L, Yendaw E. Factors correlated with low birth weight in Ghana. Br J Med Med Res 2016; 16:1-8.

15. Almeida FSS, Morrone LC, Ribeiro KB. Tendências na incidência e mortalidade por acidentes de trabalho no Brasil, 1998 a 2008. Cad Saúde Pública 2014; 30:1957-64.

16. Barbosa IR, Souza DLB, Bernal MM, Costa ICC. Desigualdades regionais na mortalidade por câncer de colo de útero no Brasil: tendências e projeções até o ano de 2030. Ciênc Saúde Coletiva 2016; 21:253-62.

17. Kim H, Fay, MP, Feuer EJ, Midthune DN. Permutation test for joinpoint regression with applications to cancer rates. Stat Med 2000; 19:335-51.

18. Ministério da Saúde. Manual de procedimentos do Sistema de Informações sobre Nascidos Vivos. Brasília: Ministério da Saúde; Fundação Nacional de Saúde; 2001.

19. Secretaria Municipal de Saúde. Pré-saber: informações de interesse à saúde. Sistema de Informações sobre nascidos vivos 2000/2001. Porto Alegre: Secretaria Municipal de Saúde; 2003.

20. Instituto Brasileiro de Geografia e Estatística. Brasil em síntese: educação. http://brasilem sintese.ibge.gov.br/educacao.html/ (acessado em 19/Ago/2016).

21. De Souza Buriol VC, Hirakata V, Goldani MZ, da Silva CH. Temporal evolution of the risk factors associated with low birth weight rates in Brazilian capitals (1996-2011). Popul Health Metr 2016; 14:15.

22. Ministério da Saúde. Pesquisa Nacional de Demografia e Saúde da Criança e da Mulher. PNDS 2006: relatório final. Brasília: Ministério da Saúde; 2008.

23. Instituto Brasileiro de Geografia e Estatística. Estatísticas do Registro Civil. Rio de Janeiro: Instituto Brasileiro de Geografia e Estatística; 2012.

24. Hernandez AR, Silva CH, Agranonik M, Quadros FM, Goldani MZ. Analysis of infant mortality trends and risk factors in Porto Alegre, Rio Grande do Sul State, Brazil, 1996-2008. Cad Saúde Pública 2011; 27:2188-96.

25. Vettore M, Lamarca G. Atenção pré-natal no Brasil: uma questão de oferta, de acesso ou de escolaridade materna? http://dssbr.org/si te $/$ ? $=10326 \&$ preview $=$ true (acessado em 05/ Mar/2014).
26. Guanabens MCFG, Gomes AM, Mata ME, Reis ZSN. Gravidez na adolescência: um desafio à promoção da saúde integral do adolescente. Rev Bras Educ Méd 2012; 36:20-4.

27. Santos NLAC, Costa MCO, Amaral MTR, Vieira GO, Bacelar EB, Almeida AHV. Teenage pregnancy: analysis of risk factors for low birth weight, prematurity and cesarean delivery. Ciênc Saúde Coletiva 2014; 19:719-26.

28. Pedraza DF, Rocha AC, Cardoso MV. Prenatal care and birth weight: an analysis in the context of family health basic units. Rev Bras Ginecol Obstet 2013; 35:349-56.

29. Mello Jorge MHP, Gotlieb SLD, Oliveira H. O Sistema de Informação sobre Nascidos Vivos: primeira avaliação dos dados brasileiros. Inf Epidemiol SUS 1996; 2:15-48.

30. Guimarães EAA, Loyola Filho AI, Hartz ZMA, Meira AJ, Luz ZMP. A descentralização do SINASC e a completude das variáveis da declaração de nascido vivo em municípios mineiros de 1998 a 2005. Rev Bras Crescimento Desenvolv Hum 2011; 21:832-40.

31. Programa das Nações Unidas para o Desenvolvimento. Brasil. http://www.undp.org/con tent/brazil/pt/home/countryinfo/ (acessado em 10/Set/2016).

32. Mishimina FC, Scochi CGS, Ferro MAR, Lima RAG, Costa IAR. Declaração de nascido vivo: análise do seu preenchimento no Município de Ribeirão Preto, São Paulo, Brasil. Cad Saúde Pública 1999; 15:387-95.

33. Guerra FAR, Llerena JJC, Gama SGN, Cunha $\mathrm{CB}$, Theme Filha MM. Confiabilidade das informações das declarações de nascido vivo com registro de defeitos congênitos no Município do Rio de Janeiro, Brasil, 2004. Cad Saúde Pública 2008; 24:438-46.

34. Marcarenhas MDM, Gomes KRO. Confiabilidade dos dados do Sistema de Informações sobre Nacidos Vivos em Teresina, Estado do Piauí, Brasil - 2002. Ciênc Saúde Coletiva 2011; 16 Suppl 1:1233-39.

35. Gabriel GP, Chiquetto L, Morcillo AM, Ferreira MC, Bazan IGM, Daolio LD, Lemos JJR, Carniel EF. Avaliação das informações das Declarações de Nascidos Vivos do Sistema de Informação sobre Nascidos Vivos (Sinasc) em Campinas, São Paulo, 2009. Rev Paul Pediatr (Online) 2014; 32:183-88.

36. Jorge MHP, Laurenti R, Gotlieb SLD. Quality analysis of Brazilian vital statistics: the experience of implementing the SIM and SINASC systems. Ciênc Saúde Coletiva 2007; 12:64354.

37. Theme Filha MM, Gama SGN, Cunha CB, Leal MC. Confiabilidade do Sistema de Informações sobre Nascidos Vivos Hospitalares no Município do Rio de Janeiro, 1999 -2001. Cad Saúde Pública 2004; 20 Suppl 1:S83-91. 


\section{Abstract}

The article assessed the quality of completion of the maternal school variable in Brazilian state capitals and its regional distribution, based on the Brazilian Information System on Live Births (SINASC) with processed data from live birth certificates. A descriptive study was conducted in the time series from 1996 to 2013, with a total de 12,062,064 births, of which 11,442,494 (94.86\%) had valid information on the maternal schooling variable. The results were calculated as the number of incomplete results in the variable per 1,000 live births, and the trend was assessed with the Joinpoint software, version 4.3.1. According to regional analysis, the South of Brazil showed a downward trend in incompleteness of maternal schooling throughout the study in all the state capitals of that region. Most of the country's other state capitals also showed improvement in the variable's completeness. However, there were different trends in some state capitals, even with greater incompleteness at the end of the period when compared to the beginning. SINASC proved to be a valuable source of data on mothers and their newborns, besides information on conditions in labor, delivery, and birth in the country. Maternal schooling, considered an important factor for obstetric and neonatal outcomes, is particularly useful for elaborating and evaluating policies and measures in maternal and child health. Thus, to achieve maximum completeness in data on this variable requires joint effort by health professionals and administrators, thereby guaranteeing the data's trustworthiness.

Birth Certificates; Educational Status; Information Systems

\section{Resumen}

El presente artículo evaluó la calidad en la cumplimentación de la variable escolaridad de la madre en las capitales brasileñas y su distribución regional, mediante el Sistema de Información sobre Nacidos Vivos (SINASC), registrado vía la Declaración de Nacido Vivo (DNV). Se realizó un estudio descriptivo de una serie temporal, durante el periodo de 1996 a 2013, con un total de 12.062.064 nacimientos, de los cuales 11.442.494 $(94,86 \%)$ contaban con información válida de la variable escolaridad de la madre. Los resultados se calcularon por número de registros con carácter incompleto de la variable por cada 1.000 nacidos vivos y se evaluó la tendencia mediante el programa Jointpoint (versión 4.3.1). El análisis regional demostró que la región sur presentó una tendencia en la reducción de la incompletitud de la escolaridad materna, sostenida durante el período del estudio, en todas sus capitales. Igualmente, de manera general, la mayor parte de las otras capitales del país también evidenció una mejora en la completitud de la variable. No obstante, se verificaron diferentes tendencias con algunas capitales, inclusive, algunas presentando una mayor incompletitud al final del período, cuando se compara con el principio del mismo. El SINASC demostró ser un instrumento valioso para la información sobre las madres y sus recién nacidos, junto a las condiciones de parto y nacimiento en el país. Particularmente, la escolaridad materna, considerada un factor importante sobre los desenlaces obstétricos y neonatales, es una variable que permite la elaboración y evaluación de las políticas y acciones en el área de la salud materno-infantil. De esta forma, alcanzar su máxima completitud requiere un esfuerzo conjunto, de profesionales y gestores, garantizando la credibilidad de esta información.

Certificado de Nacimiento; Escolaridad;

Sistemas de Información
Recebido em 07/Mar/2017

Versão final reapresentada em 07/Jun/2017

Aprovado em 27/Jun/2017 